\title{
Les trajectoires documentaires, une proposition de modèle pour analyser les interactions des enseignants avec les ressources au fil du temps: la plateforme AnA.doc, un outil d'instrumentation de cette analyse
}

\section{Documentational trajectories, a prospective model for analyzing teachers' interactions with resources over the time: the AnA.doc platform, a tool for instrumenting this analysis}

\author{
Katiane de Moraes Rocha ${ }^{1}$ a \\ ${ }^{1}$ École Normale Supérieure de Lyon \\ 46 Allée d'Italie, Lyon 69007, France
}

\begin{abstract}
Résumé. Cet article souhaite contribuer à la discussion de la question : comment le chercheur conçoit-il et traite-t-il ses données ? Nous présentons un exemple d'analyse et de traitement des données en nous appuyant sur le développement de la plateforme AnA.doc. Nous présentons ici les réflexions théoriques et méthodologiques qui ont servi de base pour créer cet outil, telles l'approche documentaire du didactique et l'investigation réflexive. Pour cela nous mobilisons l'exemple d'une enseignante de mathématiques au collège, Sophie, analysé comme une étude de cas dans le cadre d'une recherche doctorale. L'objectif de recherche est de comprendre comment les enseignants construisent leur expérience avec les ressources tout au long de leur carrière. L'analyse partielle montre comment une ressource donnée structure la pratique d'un enseignant. L'objectif de la plateforme est finalement de constituer un incubateur d'analyse du travail documentaire des enseignants.
\end{abstract}

\begin{abstract}
This article contributes to the discussion of the issue: how does the researcher design and process his / her data? We present an example of analysis and data processing based on the development of the AnA.doc platform. We present here the theoretical and methodological reflections grounding the design of this tool, such as the documentational approach to didactics and reflective investigation. For this purpose, we use the example of a high school mathematics teacher, Sophie, as a case study (which is developed as part of a $\mathrm{PhD}$ project). Our analysis aims at understanding how teachers build their experience with resources over time. This partial study evidences how a given resource structures the practice of a teacher. The objective of this platform is to set up an incubator of analyses of the work of teachers with resources.
\end{abstract}

\footnotetext{
a Auteure de correspondance : katiane.de-moraes-rocha@ens-lyon.fr
} 


\section{Introduction}

Notre proposition vise à contribuer au questionnement : comment le chercheur conçoit-il et traite-t-il ses données? Cette contribution est basée sur la présentation d'un exemple de conception et de traitement des données d'une recherche de doctorat qui se passe en France. Nous présentons la plateforme AnA.doc (Analyse de la documentation), outil utilisé par le chercheur pour partager et analyser les données relatives au travail d'un enseignant de mathématiques avec ses ressources. Ce travail dans lequel l'enseignant cherche, développe, met en œuvre et partage des ressources est nommé par Gueudet et Trouche (2008) travail documentaire. Ce travail dépasse les limites de la classe et a lieu en plusieurs endroits et en des moments différents : dans une recherche fortuite en ligne, un échange avec un collègue, la participation à une formation, la préparation d'une séance à la maison, etc. La profusion des ressources numériques a bouleversé fortement les types de ressources et d'environnements que l'enseignant utilise : forums, réseaux sociaux, sites, dossiers partagés en ligne, etc. Tout cela rend l'analyse de l'activité des enseignants plus complexe. Cependant, les ressources numériques nous fournissent aussi des nouveaux outils pour capter ce travail : enregistrements audio et/ou vidéo des acteurs en action, captation d'écrans, etc. Dans ce contexte, la question qui se pose est: comment instrumenter l'analyse des données du point de vue de la pratique de l'enseignement des mathématiques?

Cette question est aussi posée au sein du programme de recherche ReVEA ${ }^{1}$. Ce projet cherche à analyser les questions autour des usages et des productions des ressources par l'enseignant. C'est dans ce projet que la plateforme AnA.doc s'insère. C'est aussi dans ce projet qu'Anita Messaoui ${ }^{2}$ inscrit ses analyses sur le développement de l'expertise construite par l'enseignant dans son travail documentaire, qu'elles nomment expertise documentaire. Dans le cadre de notre thèse ${ }^{3}$, nous cherchons à comprendre comment les enseignants construisent leur expérience au fil du temps dans l'interaction avec ses ressources, à travers le développement des notions d'expérience et de trajectoire documentaire.

Cet article est partagé en quatre sections. Nous présentons d'abord le cadre théorique qui est la base, à la fois de la création de la plateforme, et de notre travail de recherche avec l'enseignant. Ensuite, nous présentons quelques choix méthodologiques pour le suivi de l'enseignant. Puis, nous présentons les éléments principaux de la plateforme. Après, nous nous appuyons sur un exemple d'analyse du travail documentaire d'un enseignant pour montrer le fonctionnement, les potentialités et les contraintes de cette plateforme pour l'analyse des données. Et pour conclure, nous présentons nos considérations finales en discutant nos choix méthodologiques, les avantages et les contraintes dans l'utilisation de la plateforme.

\section{Référentiel théorique}

Dans cette section, nous présentons quelques concepts clés de l'approche documentaire du didactique : ressource, système des ressources, documents et schèmes (Gueudet et Trouche, 2008). Nous traitons aussi d'autres concepts qui nous permettent de traiter les questions

1 ANR ReVEA, Ressources vivantes pour l'enseignement et l'apprentissage, 2014-2018 (https://www.anr-revea.fr/).

${ }_{3}^{2}$ En écho à l'article d'Anita Messaoui présent dans ces actes d'ICODOC 2017.

${ }^{3}$ Thèse dirigée par Luc Trouche à l'ENS de Lyon, Laboratoire S2HEP, École doctorale EPIC, bourse CNPq. 
autour des usages de ressources par l'enseignant (Prieur, 2016 ; Ruthven, 2010 ; Vergnaud, 1994). Pour finir, nous présentons les concepts d'expérience et de trajectoire documentaire. L'approche documentaire du didactique développée par Gueudet et Trouche (2008) met en lumière le travail des enseignants avec les ressources au cœur de leur activité professionnelle. En effet, c'est l'enseignant qui est responsable de créer et mettre en œuvre ses ressources. Le terme ressource est défini par les auteurs à partir du travail d'Adler (2000). Selon elle, la ressource est tout ce qui re-source le travail de l'enseignant. Le terme ressource désigne donc tout ce que l'enseignant utilise pour réaliser son travail, par exemple une idée qu'un collègue a proposée, une photocopie, un logiciel, un manuel scolaire, entre autres. Ces ressources utilisées ou créées font partie d'un ensemble de ressources d'enseignement qui est dénommé système de ressources. Ce système de ressource est très complexe et nous mettons en évidence ici quatre aspects importants :

- l'entité ressource peut être explicitable par l'enseignant et différents enseignants peuvent utiliser les mêmes ressources. Cependant, une même ressource peut être utilisée de différentes façons par deux enseignants. Chaque sujet élabore ses connaissances par rapport à la ressource, la transforme en ce que Gueudet et Trouche (2008) nomment un document. Ainsi, le document est une entité mixte formée par les ressources du sujet ainsi que par les connaissances qui y sont associées ;

- les connaissances d'un enseignant sont construites dans et pour son action. Cette action suppose un ensemble de tâches à accomplir par l'enseignant. L'enseignement de la trigonométrie en $6^{\text {ème }}$, la recherche de ressources pour l'accompagnement des élèves, la préparation d'une évaluation de géométrie ou de trigonométrie sont des exemples parmi d'autres. La résolution d'une tâche s'inscrit dans le cadre d'une situation particulière. Cependant, certaines tâches sont voisines (par exemple, préparer une évaluation de géométrie et une de trigonométrie); ce qui va conduire l'enseignant à élaborer une organisation pour accomplir ces types des tâches. Ces tâches voisines vont composer ce que Vergnaud (1994) désigne par classe de situations. Cette organisation qui englobe les connaissances élaborées par le sujet est nommée schème. Ces situations dans le cas de l'enseignant sont des situations de travail comme par exemple, préparer une leçon, mettre en œuvre une leçon, etc.... Le schème est, ainsi, défini par Vergnaud (1994:180) comme « une totalité dynamique fonctionnelle, c'est-à-dire quelque chose qui fonctionne comme une unité... c'est une organisation invariante de la conduite pour une classe de situations données ... »;

- des ressources peuvent avoir des statuts différents. Comme l'a montré Prieur (2016), certaines ressources rassemblent des principes qui guident la conception des ressources, elle les nomme métaressources. Ce type de ressource soutient une posture réflexive du travail documentaire de l'enseignant et «intègre un niveau d'explicitation qui correspond à des descriptions et des justifications du travail documentaire ou encore des prévisions sur les usages des ressources produites » (Prieur, 2016:67);

- l'intégration et la conception de certaines ressources structurent la pratique de l'enseignement en classe. Ruthven (2010) présente cinq caractéristiques structurantes de la pratique de l'enseignant :

(1) l'environnement de travail qui comprend tous les aspects matériels autour de l'endroit où le cours se réalise ;

(2) le format d'activité qui concerne les modèles d'interaction entre les élèves et l'enseignant (routines, règles, etc.) ;

(3) le système de ressources qui, selon l'acteur, inclut l'ensemble des outils et supports mathématiques de la classe ;

(4) l'économie temporelle qui est l'équilibre entre le temps d'enseignement et le temps d'apprentissage des élèves (appelé aussi temps didactique) ; 
(5) le script curriculaire qui est considéré au sens cognitif comme une forme d'organisation structurée de l'activité finalisée, et que nous analyserons en relation avec le concept de schème.

Le travail documentaire est continu tout au long de la vie professionnelle de l'enseignant, et au fil du temps, les enseignants font face à différentes situations qui les amènent à développer leurs documents pour enseigner les mathématiques : préparer une leçon suite à un changement de programme, participer d'une formation, etc.

Notre analyse du développement professionnel d'un enseignant se centre sur l'analyse de son activité, suivant Pastré (2011: 48) dans le cadre de la didactique professionnelle: « une centration sur l'activité au sens large, c'est-à-dire incluant l'apprentissage ; [...] une centration sur l'importance de la dimension cognitive présente dans l'activité notamment sous la forme de la conceptualisation dans l'action». Dans ce cadre, le sujet est considéré comme quelqu'un qui s'adapte, au fil du temps, en faisant face à plusieurs situations. Et le développement est compris «comme [la] construction de soi, comme appropriation de l'ensemble des événements vécus par un sujet pour leur donner du sens pour soi » (Pastré, 2011 : 118). Nous émettons l'hypothèse que, au long de leur carrière, les enseignants rencontrent certains événements professionnels qui ont un rôle marquant dans leur travail documentaire en impactant leur façon de produire et d'utiliser leurs ressources. Et c'est à travers ces événements que les enseignants vont construire leur expérience. Nous reprenons le terme expérience de Pastré (2011), qui le définit comme l'accumulation et l'appropriation du passé par le sujet. Nous allons nommer l'expérience construite à travers des interactions avec les ressources l'expérience documentaire d'un enseignant. Nous considérons les événements professionnels dans un sens très large, comme tout ce qui est identifié par le chercheur ou par l'enseignant comme ayant une conséquence sur le travail documentaire de cet enseignant. Nous allons nommer l'ensemble des événements identifiés de trajectoire documentaire (TD) de l'enseignant. Le repérage de ces événements nous donne des indices concernant les vecteurs de développement professionnel de cet enseignant.

\section{Cadre méthodologique}

Dans cette section, nous présentons d'abord nos principes et outils méthodologiques. Ensuite, nous abordons la plateforme AnA.doc et notre questionnement de recherche.

\subsection{Principes et outils méthodologiques}

Nous présentons ici les principes de l'investigation réflexive (Gueudet \& Trouche, 2010) qui ont inspiré nos choix méthodologiques et la création de la plateforme. Nous abordons aussi quelques outils employés dans notre suivi des enseignants.

L'investigation réflexive proposée par Gueudet et Trouche (2010:61) rassemble quatre principes importants pour suivre le travail documentaire d'enseignant :

(1) durée longue du suivi, car les genèses sont développées sur le long terme; le temps est donc primordial pour analyser la stabilité et les évolutions du travail de l'enseignant ;

(2) suivi en tout lieu, en cherchant à accompagner le travail documentaire dès sa préparation jusqu'à sa mise en œuvre, cela concerne aussi le travail hors établissement ;

(3) recueil étendu des ressources matérielles utilisées et produites par l'enseignant pour garder les traces du travail documentaire d'enseignant; relatives aux ressources créées ou mobilisées par l'enseignant ; 
(4) suivi réflexif, c'est-à-dire promouvant la réflexion des enseignants sur leur travail documentaire, cela nous permet de mieux comprendre comment et pourquoi l'enseignant s'approprie les ressources de son enseignement.

Dans cette perspective, nous avons conçu nos outils méthodologiques, qui peuvent être présentés en trois catégories : observer l'enseignant en action, le suivre à distance et réaliser des entretiens avec l'enseignant suivi. Pour l'analyse de l'enseignant en action, nous avons recueilli les données de deux moments cruciaux pour comprendre son travail documentaire : la préparation d'une séance et la mise en œuvre d'une séance.

Pour accompagner le travail d'enseignant en tous lieux, un suivi à distance vise à compléter les données obtenues à travers de l'observation de l'action. Pour cela nous avons employé deux outils : la boîte réflexive et le journal de bord. La boîte réflexive est un dossier en ligne sur un espace partagé avec l'enseignant où le chercheur peut poser des questions, solliciter et consulter les ressources que l'enseignant exploite et/ou produit. Le journal de bord, où l'enseignant consigne des événements marquant de son travail documentaire, a été utilisé pour repérer des événements qui ont eu des relations avec le travail documentaire de l'enseignant.

Concernant la dernière catégorie, nous avons mis en œuvre plusieurs entretiens qui visent à faire réfléchir les enseignants sur leur travail documentaire. Nous avons proposé, par exemple, que les enseignants créent des cartographies réflexives (Rocha, 2018) soit de leur système de ressources, soit de leur trajectoire documentaire.

Pour le développement de tous ces outils, nous utilisons différents médias : enregistrement en audio et en vidéo captant l'activité de l'enseignant, vidéo captant l'écran de son ordinateur, photo, digitalisation des ressources sur support papier, entre autres. La plateforme AnA.doc nous a fourni un instrument de stockage, de traitement et de partage de ces données hétérogènes (vidéos, audio, photos, images ou encore textes) que nous présentons dans la section ci-après.

\subsection{Présentation de la plateforme AnA.doc et du cas d'étude}

La plateforme a été créée pour servir de support à l'analyse de la variété des situations du travail documentaire d'un enseignant. Les chercheurs intéressés par l'analyse de ce travail constituent son principal public. Elle rend possible (i) le stockage de données de différentes natures (vidéo, images, audio, etc.), (ii) la création d'analyses mobilisant ces données et (iii) les interactions entre les chercheurs. Ce dernier aspect est très important, car une interaction entre les chercheurs est l'objectif central de son utilisation. En effet, un chercheur qui visite la plateforme peut poser des questions ou même proposer une analyse sur le corpus de données d'un autre chercheur.

La plateforme contient trois niveaux: un niveau de situations décrites suivant un modèle donné, et accompagnées des ressources en jeux ; un niveau de webdocuments qui proposent des analyses d'une (ou des) situation(s), en s'appuyant sur les données qu'elles intègrent ; un niveau de glossaire visant une compréhension partagée des termes utilisés, où les chercheurs présentent les notions en fonction des cadres théoriques utilisés.

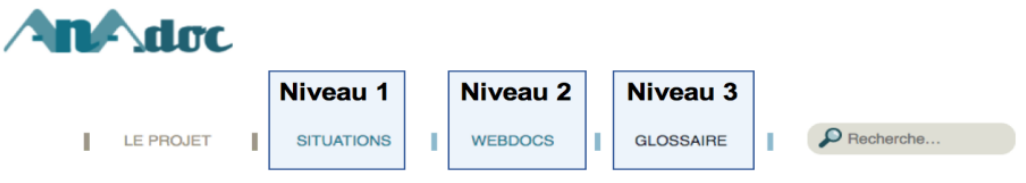

Figure 1. Structure de la plateforme AnA.doc 
Dans la plateforme, chaque situation contient un descriptif pour préciser les aspects méthodologiques et la problématique de la recherche : les aspects généraux de la situation, une présentation du sujet et de son contexte, les intérêts de la recherche, les méthodes employées, les liens avec la situation et les données relatives à la situation. La plateforme présente des situations séparées en quatre types: analyse réflexive, conception des ressources, mises en œuvre et révision d'une séance Nous traitons dans cet article un exemple d'analyse de la situation de type «analyse réflexive» qui est intitulé «Sophie (mathématiques, collège), évoque son expérience professionnelle». Dans la Figure 2, nous présentons une partie de la description de la situation, ce qui nous permet aussi de présenter notre sujet de recherche.
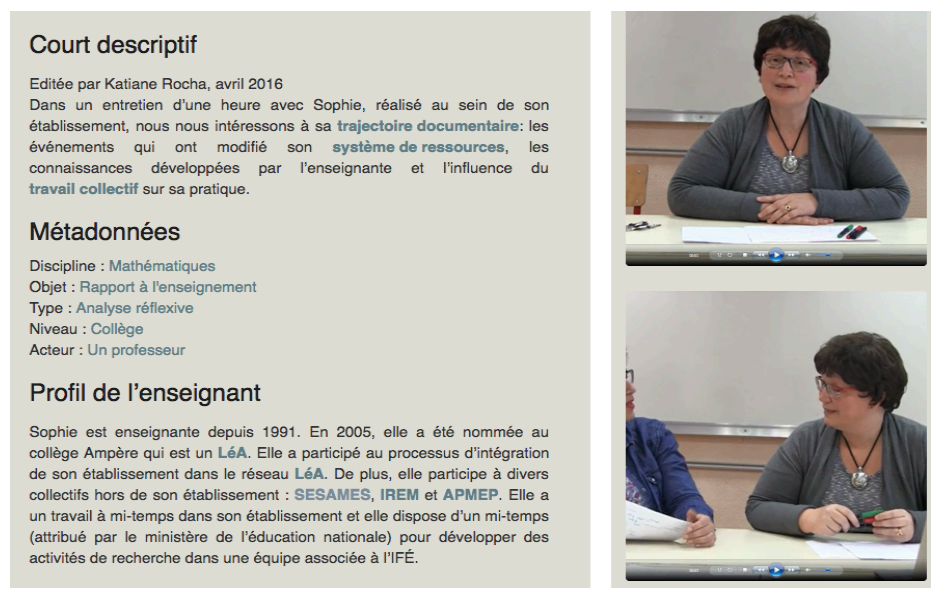

Figure 2. Une situation de préparation d'une leçon par Sophie ${ }^{4}$

Dans AnA.doc, contrairement à une situation (niveau 1) qui cherche à présenter les données de manière exhaustive, autant que faire se peut, le webdocument (niveau 2) propose l'analyse d'une question précise sur le travail documentaire d'un enseignant. Par exemple, dans cet article, nous présentons le webdocument intitulé "L'influence d'une métaressource sur la trajectoire documentaire de Sophie (mathématiques, collège)». L'analyse suit une structure similaire à celle d'un un écrit qui appartiendrait à la catégorie "article universitaire", c'est-à-dire qu'elle contient un cadrage qui présente les aspects théoriques évoqués, une analyse, une conclusion et des références. Chaque inférence de recherche du chercheur s'appuie sur un petit nombre d'illustrations (extraits vidéo, images, graphiques, etc.) extraites du corpus de données, illustrations les plus concises possibles (des extraits vidéos de moins de $3 \mathrm{mn}$ par exemple ${ }^{5}$ ). Un webdocument constitue ainsi un outil pour développer une première analyse d'une situation en fonction d'une question précise. Il donne accès à un forum, qui permet à d'autres chercheurs de commenter ou critiquer l'analyse proposée, à partir des extraits choisis, ou en revenant à l'ensemble du corpus rassemblé au niveau de la situation elle-même. Un webdocument, finalement, constitue un incubateur d'analyses du travail documentaire d'un enseignant donné. Nous

\footnotetext{
${ }^{4}$ Tous les termes en bleu et gras sont définis dans le glossaire de AnA.doc qui permet à une communauté de chercheurs de préciser le sens des termes utilisés dans les descriptions et les analyses. Ici nous présentons seulement les sigles: (IREM) Instituts de Recherche sur l'Enseignement des Mathématiques, (SESAMES) Situations d'Enseignement Scientifique : Activités de Modélisation, d'Évaluation, de Simulation, (APMEP) Association des Professeurs de Mathématiques de l'enseignement Public et (Léa) Lieux d'éducation associées à l'Institut Français de l'Éducation.

${ }^{5}$ AnA.doc intègre un outil permettant de pointer directement sur le début et la fin d'une vidéo intégrée dans la situation en question.
} 
présentons dans la prochaine section quelques éclairages sur le webdocument que nous avons présenté ci-dessus.

\section{Un exemple d'analyse dans la plateforme : le webdocument}

Ce webdocument explore une ressource en particulier dans le travail de Sophie intitulée la Mise En Train (MET). Le mot «train» est un acronyme qui signifie « Travail de Recherche d'Approfondissement avec prise d'INitiative» qui consiste à un ensemble d'activité à donner aux élèves au début d'heure de classe pour travailler une notion. Dans cette section, nous situons cette ressource dans la trajectoire documentaire de Sophie. Ensuite, nous montrons que la MET est une métaressource, car elle guide une partie de son travail documentaire (Prieur, 2016). Nous montrons en quoi elle structure son travail documentaire. Cette entrée dans la TD à travers la métaressource MET soutient le questionnement suivant: «comment une métaressource agit-elle sur la trajectoire documentaire de Sophie?».

\subsection{Analyse}

La ressource MET a été identifiée dans la Cartographie réflexive de la trajectoire documentaire $^{6}$ réalisée par Sophie (Figure 3). Elle est apparue associée à l'événement «Partenariat avec Cindy» à travers les deux articles qu'elles ont rédigés pour diffuser cette ressource. Elle a été créée au sein du collectif Sésames ${ }^{7}$. MET est une façon de concevoir des ressources en amont et de réfléchir à la façon dont les élèves travaillent au début de chaque séance. Chaque heure de classe est ainsi partagée en deux : le moment MET et le moment cours. Durant la MET, il ne s'agit pas de réaliser des exercices sur le contenu travaillé, mais de créer plusieurs entrées pour construire un concept mathématique ou pour repérer des connaissances déjà travaillées.

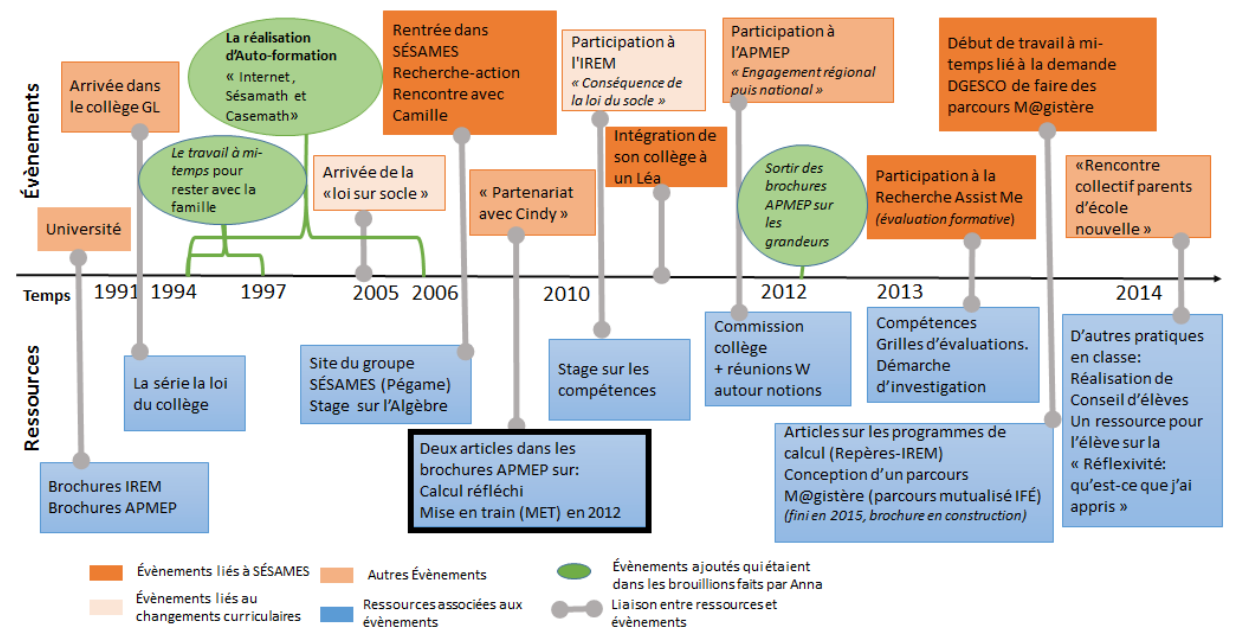

Figure 3. Transposition numérique de la cartographie réflexive de la trajectoire documentaire de Sophie

${ }^{6}$ Dans cette figure, sont représentés les événements professionnels (au-dessus de la ligne du temps), identifiés par l'enseignant comme ayant des conséquences sur son travail documentaire, et les ressources associées (au-dessous de la ligne du temps).

${ }^{7}$ Site : http://pegame.ens-lyon.fr/. 
Pendant l'entretien de la situation, Sophie nous a expliqué en quoi consiste la MET. Nous présentons dans le webdocument un extrait vidéo d'environ 2 min de cette explication. Cidessous, nous avons transcrit quelques extraits dans lesquels l'enseignante met en évidence des aspects importants qui guident la production de ressources MET : mettre les élèves au travail dès qu'ils rentrent en classe, 'chauffer' (au sens sportif) les élèves, rentrer progressivement dans un problème, favoriser la recherche par les élèves.

Extrait 1: "C'était vraiment l'idée, c'était comment gérer ce temps pour que le professeur au début de l'heure, il puisse faire son appel, vérifier les devoirs, vérifier s'il y a des absences ou pas, etc., tranquillement pendant que les élèves eux ils sont en train de travailler, pas en attendant, pas que les élèves attendent que le professeur fait tout ce qui est administratif pour pouvoir commencer. (...) on ne voulait plus faire trois heures à réviser la distributivité par exemple. Donc, on se disait que faire par petites touches ah, ça pourrait être intéressant, ils n'étaient pas... (...) on s'est rendu compte que les élèves en fait, bon à part les premiers mois qu'ils sont tout déstabilisés, mais ensuite, ils étaient plutôt contents d'avoir un énoncé et de ne pas savoir de quoi ça allait parler, et d'avoir la surprise et de chercher du coup $»$.

Nous pouvons noter qu'il y a certains principes au cœur de la conception des activités de MET. Ces principes vont guider la production des ressources par l'enseignant; autrement dit, la MET est une métaressource pour l'enseignant. En effet, Sophie change la façon de concevoir les ressources, notamment la progression qui est partagée en deux colonnes: cours principal et MET. Cette évolution offre trois possibilités pour aborder un thème donné. La première est de commencer à traiter un thème dans la partie MET et de l'inclure dans un deuxième temps dans le cours principal. La deuxième est de commencer à travailler un thème dans la partie cours et après le passer à la partie MET. Et la troisième et dernière option consiste à exploiter un thème complètement dans la partie MET.

Dans l'extrait 2, Sophie mentionne que la ressource qui organise le cours par thème a aussi changé, car elle contient aussi des alertes pour les MET liées aux thèmes. Nous présentons en bas de l'extrait une séquence d'images de cet extrait vidéo, elle montre des détails qu'on peut voir dans cet extrait : comment elle cartographie son système de ressources, les gestes pendant qu'elle explique et les fenêtres affichées sur son écran d'ordinateur pour nous présenter les ressources qu'elle mobilise.

Extrait 2 : "Ça c'est les ressources comme ça, sinon quand on va dans un chapitre des cours, les Mises en train apparaissent. Un chapitre des cours peut-être de troisième, non pas cette année. Dans nos ressources, si je prends par exemple calcul littéral [elle cherche]. Si je prends...Si je prends mon cours, je vais avoir à chaque fois les programmes et puis derrière comment $j$ 'ai leur répartis je vais avoir les Mise en train. (...) Le chapitre il vit autrement, parce qu'il va falloir - et là je ne vais pas trouver comme ça - mais il y a des chapitres qui vont être marqués "attention il va falloir faire avant telle Mise en train, telle Mise en train, telle Mise train". Donc, quand on a un chapitre, ces choses-là, elles sont relues ».

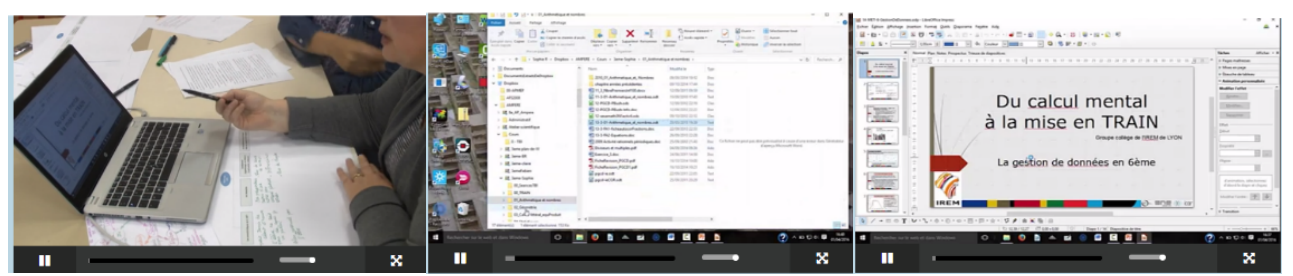

Figure 4. Copies d'écran de l'extrait vidéo 
Le système de ressources est ainsi transformé et adapté. Nous pouvons observer l'intégration des activités de la MET dans la progression et dans le cours, mais aussi (i) la création d'un bilan personnel des élèves qui est spécifique à la MET, dans laquelle ils vont mettre toutes les activités sur lesquelles ils ont travaillé à ce moment-là ; (ii) la création des nouvelles ressources qui sont mises à disposition dans le site Pégame du groupe Sésames (groupe dans lequel la MET a été développé); (iii) la création de ressources pour organiser les activités MET, des diaporamas (Figure 4) qui rassemblent plusieurs entrées possibles d'un même thème (fonction, racine carrée, programme calcul, fractions, entre autres) ; (iv) la création des séquences des activités à traiter une classe dans une ressource séparée par niveau.

Nous avons pu observer quelques éléments qui nous montrent les transformations dans le système de ressource de Sophie, mais aussi dans le format d'activité en classe, puisque de nouvelles routines sont créées entre les élèves et l'enseignant. De même, l'environnement de travail change lorsque les élèves arrivent en classe en retard. Ils échangent avec leurs voisins pour savoir quelle activité la classe est en train de réaliser. Nous attirons par ailleurs l'attention sur l'économie temporelle. Cette caractéristique est en effet cruciale pour comprendre le rôle de la métaressource dans le travail en classe : dans un premier temps, les élèves n'ont pas conscience des concepts mathématiques qui sont en jeu ; dans un deuxième temps, ils développent des connaissances tout au long de la mise en œuvre de la MET; et dans un troisième temps, ils mobilisent ces connaissances pour conjecturer et résoudre les problèmes... Nous pouvons voir à travers des ressources produites par Sophie que la création de la MET a été aussi un processus de genèse des connaissances, que nous allons exploiter dans d'autres cas. Nous pouvons souligner que dans le webdocument, nous avons fourni un extrait de vidéo pour signaler les transformations de chaque caractéristique structurante de la pratique d'enseignant.

La métaressource MET a été conçue et formalisée au sein de discussions collectives dans le cadre du groupe SESAMES. Elle a dépassé l'objectif initial d'organisation du temps de classe : elle a fourni une façon de concevoir les ressources et de travailler avec les élèves. Nous ne l'avons pas traité ici, mais cette ressource utilisée par Sophie est partagée au sein de plusieurs collectifs (IREM, APMEP, collège de Sophie, etc.). La création des MET a engendré des mutations dans son travail documentaire à travers la production de nouvelles ressources. Il s'agit bien d'un développement professionnel : ouverture à de nouvelles ressources, partage d'expertise dans des collectifs, une évolution de la perception du métier d'enseignant. Par ailleurs, cette métaressource structure plusieurs ressources utilisées directement en classe (progression, séquences, etc.). D'une manière plus générale, nous avons montré que la "Mise en train" agit sur chaque caractéristique structurante présentée par Ruthven (2010). Ces évolutions sont le signe d'un développement professionnel de Sophie, bien marqué dans sa trajectoire documentaire.

\section{Considérations finales et perspectives}

Pour finir, nous revenons sur la question proposée dans l'axe 3 : comment le chercheur conçoit-il et traite-t-il ses données? Ce que nous avons fait est une articulation entre le cadre méthodologique et la prise des données : investigation réflexive et données diverses. Nous avons exploité les potentialités dans l'utilisation des différents médias pour rendre compte de processus complexes et dynamiques, comme le travail documentaire, dont l'analyse suppose une prise en compte d'un grand nombre de données de différentes. Nous avons comme hypothèse que la plateforme peut faciliter le partage du processus de recueil et d'analyse des données. Ce partage est très enrichissant pour la recherche, car l'on peut échanger différents points de vue d'analyse. L'interaction entre les chercheurs pour 
construire cette analyse nous amène à une validation collective des résultats tout au long du processus.

La plateforme a quelques contraintes : la première est liée au moment de saisie les données qui exige beaucoup de temps pour les décrire ; la deuxième la partie de partage des données entre divers communautés n'a pas été encore travaillé ; le dernier, ce n'est pas possible de faire l'exportation des webdocuments pour autres formats. Cependant, AnA.doc constitue une instrumentation productive, intégrant un ensemble d'outils et soutenant des interactions très riches avec d'autres chercheurs (pour une analyse plus approfondie, voir Alturkmani et al., à paraître).

\section{Bibliographie}

Adler J. (2000). Conceptualising resources as a theme for teacher education. Journal of Mathematics Teacher Education 3, p. 205-224.

Alturkmani M., Daubias P., Loisy C., Messaoui A., Trouche L. (à paraître). Instrumenter les recherches sur le travail documentaire des enseignants : le projet AnA.doc. Education \& didactique.

Gueudet G. et Trouche L. (2008). Du travail documentaire des enseignants : genèses, collectifs, communautés. Le cas des mathématiques. Education \& didactique, vol. 2, n³, p. 7-33.

Gueudet G. et Trouche L. (dirs.) (2010). Ressources vives. Le travail documentaire des professeurs en mathématiques. Rennes : PUR.

Pastré P. (2011). La didactique professionnelle. Approche anthropologique du développement chez les adultes. Paris : Presses universitaires de France.

Prieur M. (2016). La conception codisciplinaire de métaressources comme appui à l'évolution des connaissances des professeurs de sciences. Thèse de doctorat: Université Claude Bernard Lyon I, https://hal.archives-ouvertes.fr/tel-01364778v1

Rocha K. (2018). Uses of online resources and documentational trajectories: the cases of Sésamath. In L. Fan, L., Trouche, C. Qi, S. Rezat, \& J. Visnovska, J. (Eds), Research on Mathematics Textbooks and Teachers' Resources: Advances and issues. Berlin : Springer, p. 235-260.

Ruthven K. (2010). Constituer les outils et supports numériques en ressources pour la classe. In G. Gueudet et L. Trouche (dirs.), Ressources vives : Le travail documentaire des professeurs en mathématiques. Rennes : PUR, p. 183-199.

Vergnaud G. (1994). Le rôle de l'enseignant à la lumière des concepts de schème et de champ conceptuel. In M. Artigue, R. Gras, C. Laborde, \& P. Tavignot (Dir.), Vingt ans de didactique des mathématiques en France, Grenoble: La Pensée sauvage, p. 177-191. 